This item was submitted to Loughborough's Research Repository by the author.

Items in Figshare are protected by copyright, with all rights reserved, unless otherwise indicated.

\title{
Fumarate-loaded electrospun nanofibers with anti-inflammatory activity for fast recovery of mild skin burns
}

PLEASE CITE THE PUBLISHED VERSION

http://dx.doi.org/10.1088/1748-6041/11/4/041001

PUBLISHER

(C) IOP Publishing

VERSION

AM (Accepted Manuscript)

LICENCE

CC BY-NC-ND 4.0

\section{REPOSITORY RECORD}

Romano, Ilaria, Maria Summa, Jose A. Heredia-Guerrero, R. Spano, Luca Ceseracciu, C. Pignatelli, Rosalia Bertorelli, Elisa Mele, and Athanassia Athanassiou. 2016. "Fumarate-loaded Electrospun Nanofibers with Antiinflammatory Activity for Fast Recovery of Mild Skin Burns". figshare. https://hdl.handle.net/2134/23183. 


\title{
Fumarate-loaded electrospun nanofibers with anti-inflammatory activity for fast recovery of mild skin burns
}

\author{
I Romano $^{1 *}$, M Summa ${ }^{2}$ J A Heredia-Guerrero ${ }^{1}$, R. Spanò ${ }^{2}$, L Ceseracciu ${ }^{1}$, C \\ Pignatelli $^{1}$, R Bertorelli ${ }^{2}, \mathrm{E} \mathrm{Mele}^{1,3}$ and A Athanassiou ${ }^{{ }^{*}}$. \\ 1 Smart Materials, Nanophysics, Istituto Italiano di Tecnologia, Via Morego 30, 16163, \\ Genoa, Italy. \\ 2 PharmaChemistry Facility, Drug Discovery and Development, Istituto Italiano di \\ Tecnologia, Via Morego 30, 16163, Genoa, Italy. \\ 3 Department of Materials, Loughborough University, Loughborough, Leicestershire, LE11 \\ 3TU, United Kingdom. \\ E-mail: *ilaria_romano@ymail.com ; \\ *athanassia.athanassiou@iit.it
}

Keywords: polypropylene fumarate, electrospinning, wound dressings, anti-inflammatory activity.

\begin{abstract}
In the biomedical sector the availability of engineered scaffolds and dressings that control and reduce inflammatory states is highly desired, particularly for the management of burn wounds. In this work, we demonstrate for the first time, to the best of our knowledge, that electrospun fibrous dressings of poly(octyl cyanoacrylate) (POCA) combined with polypropylene fumarate (PPF) possess anti-inflammatory activity and promote the fast and effective healing of mild skin burns in an animal model. The fibers produced had an average diameter of $(0.8 \pm 0.1) \mu \mathrm{m}$ and they were able to provide a conformal coverage of the injured tissue. The application of the fibrous mats on the burned tissue effectively reducedof around $80 \%$ in the first 48 hours the levels of pro-inflammatory cytokines in comparison with not-treated animals, and enhanced skin epithelialization.Specifically, the skin appeared similar to naïve animals in term of thickness (no burn: $13.7 \pm 1.4 \mu \mathrm{m}$ versus burn+POCA:PPF: $14.3 \pm 2.5 \mu \mathrm{m}$ ), and dermal cells (no-burn $1100 \pm 112$ cells $/ \mathrm{mm}^{2} v s$. burn+POCA:PPF $1358 \pm 255$ cells $/ \mathrm{mm}^{2}$ ). Furthermore, the reduction of the epidermis thickness and cell density was of around 99and 85\%, respectively, compared to that of the injured untreated animals. Altogether, the results demonstrate the suitability of the dressing in accelerating and effectively promoting the burn healing process.
\end{abstract}

\section{Introduction}

In the last years there has been a growing research interest in new functional materials that can be applied in the biomedical field.[1,2] In particular, in the regenerative medicine area, the development of advanced systems for the efficient treatment of skin wounds is becoming crucial in order to face global demographic issues (ageing population, obesity and diabetes). $[3,4]$

Among the different types of wounds, skin burn is considered one of the worst trauma.[5] Typically, this kind of injury is treated by the following main actions: prevention and control of possible infections, absorption of fluids, modulation of hypermetabolic response, wound care and surgery.[5] Depending on the degree of burns, there are different approaches of management that can be adopted, ranging from the promotion of spontaneous re-epithelialization using powders, creams or dressings (for superficial and epidermal burns) to the application of autologous skin grafts (for full-thickness burns).[6] In all the cases, the 
availability of dressings that protect the wound site against external stresses, allow gas ex-changes, and help in restoring the normal tissue physiology by controlling the inflammatory response is highly required.[7] It has been demonstrated that fibrous dressings produced by electrospinning bioactive materials possess the potentiality to meet these requirements, thanks to their mechanical flexibility, high exposed surface area, controlled porosity, and engineered chemical properties.[8,9]

Typically, electrospun nanofibers produced forburn treatments are loaded with active agents, such as natural compounds, essential oils or drugs.[10,11]Derivatives of fumaric acid, such as monomethyl fumarate (MMF), dimethyl fumarate (DMF) and polypropylene fumarate (PPF), are also attractive materials in wound healing for their anti-inflammatory activity. MMF and DMF as well as PPF, have been already proposed for biomedical purposes.[10,11] In particular, MMF and DMF can be used for the treatment of human immunodeficiency virus (HIV) because they induce a modulation of immuno and antioxidant responses.[14] This property has been studied by Gill and Kolson[14] in order to limit the HIV disease progression. In fact, the active metabolite of the DMF and MMF inhibits the proinflammatory cytokine signaling,[14] reducing HIV infection and suppressing neurotoxin production, that drives at the neurodegeneration.[15] Furthermore, DMF has been investigated as anti-inflammatory drug in psoriasis,[12,16] and it has showed promising results in multiple sclerosis treatments.[17] On the other hand, PPF is typically used as photocrosslikable polymer for the production of three dimensional scaffolds by stereolithography[18-22] with application as bone graft substitutes.[23] An injectable PPF copolymer that guides the regeneration of many of the long bones in rats and goats has recently developed.[22] Injectable ophthalmic drug delivery systems based on PPF have been also tested as biodegradable drug carriers for long-term release of fluocinolone acetonide (FA) for ocular treatments.[23,24] Nevertheless, to the best of our knowledge, the anti-inflammatory properties of fumarates have not been yet exploited in combination with the electrospinning technique for the production of advanced fibrous systems for wound care.

Here we demonstrate that electrospun nanofibers of poly(octyl cyanoacrylate) (POCA), a biomedical polymer[25], combined with PPF are effective for the treatment of skin burns. POCA finds applications in drug delivery and targeting system, particularly for corneal and retinal surgery, and suturing internal surgery.[26] The combination of POCA and PPF allowed us to produce electrospun dressings to be used in an animal model of mild skin burns induced by ultraviolet B (UVB) light. We observed that the POCA:PPF dressings exhibited a strong anti-inflammatory activity, as proved by the analysis of pro-inflammatory cytokines and myeloperoxidase (MPO) activity. In fact, the levels of cytokines production were highly reduced in animals treated with the fibrous dressings compared to the non-treated animals (around 80\%). Furthermore, histological investigations confirmed the active effect of the fibrous dressings in preventing the progression of the inflammation. The properties of the here-discussed POCA:PPF fibres make them attractive as biomedical devices for the treatment of cutaneous inflammatory states.

\section{Experimental methods}

\subsection{Polymer solutions for electrospinning}

2-octyl cyanoacrylate (OCA) was purchased by Chenso Inc., Chemical Engineering Solution. OCA was mixed with dimethylacetamide (DMAc) (Sigma Aldrich) at 1:1 ratio (v/v) to promote the gel formation and the polymerization of the adhesive.[27] Then the resulting gel was dissolved in chloroform from Sigma Aldrich with a POCA: $\mathrm{CHCl} 3$ ratio of $1: 4(\mathrm{v} / \mathrm{v})$. PPF was synthesized following the procedure reported in Ref. 27.[28] Briefly, the fumaric acid (Sigma Aldrich) was heated in excess of propylene glycol (Sigma Aldrich) at $145{ }^{\circ} \mathrm{C}$, with overhead mechanical stirrer. A Barrette trap was connected beneath the condenser and water was collected as by-product. After 16-17 hours of reaction the temperature was increased to 180 
${ }^{\circ} \mathrm{C}$ for 4-5 hours and then it was cold down at room temperature. PPF was then dissolved in dichloromethane (Sigma Aldrich), and the solvent and the impurities were removed by rotary evaporation, for three times.[28] PPF was dissolved in chloroform with a PPF: $\mathrm{CHCl}_{3}$ ratio of $1: 4(\mathrm{v} / \mathrm{v})$. POCA and PPF solutions in chloroform were mixed at a ratio of 7:3 (v/v) and after stirring the POCA:PPF solution was used for the electrospinning technique. PCL (14 kDa) was purchased by Sigma Aldrich and was dissolved in chloroform with a ratio PCL: $\mathrm{CHCl}_{3}$ of 1:10 (w/v). As before, POCA and PCL solutions were mixed in ratio 7:3 in order to be used for the electrospinning.

\subsection{Electrospinning process}

The POCA:PPF (or POCA:PCL) solution was injected through a stainless steel 23 -gauge needle by a syringe pump (NE-1000, New Era Pump Systems Inc.) working at a flow rate of $1.8 \mathrm{~mL}$ h-1. Fibers were electrospun by applying a voltage of $15 \mathrm{kV}$ (through a high voltage power supply EH40R2.5, Glassman High Voltage Inc.) between the needle and an aluminum plate, placed at a relative distance of $15 \mathrm{~cm}$.

\subsection{Electrospun fibers characterization}

Morphological, mechanical and chemical analyses were performed on the collected POCA:PPF fibers. The morphology was investigated by a JEOL JSM-6490LA scanning electron microscope (SEM) working in high vacuum mode, with an acceleration voltage of $15 \mathrm{kV}$. It was required a coating of $10 \mathrm{~nm} \mathrm{Au} / \mathrm{Pd}$ in order to prevent charging effects. The mechanical properties of the scaffolds were characterized by uniaxial tension tests on a dual column universal testing machine (Instron 3365). Mats were cut in prismatic specimen with a width of $4 \mathrm{~mm}$ and an effective length of $25 \mathrm{~mm}$. In order to avoid accidental damage during handling, specimens were first taped on custom made paper frames composed by two detachable halves, then mounted on the measuring machine's hydraulic clamps. Displacement was applied with the rate of $1 \mathrm{~mm} / \mathrm{min}$; from the resulting stress strain curves, the Young's modulus, ultimate strength and elongation at fracture were extracted. Measurements were performed on 5 samples.

The chemical properties of POCA:PPF fibers were investigated by an attenuated total reflectance (ATR) accessory (MIRacle ATR, PIKE Technologies) equipped with a ZnSe crystal and coupled to Fourier transform infrared (FTIR) spectrometer (Equinox 70 FT-IR, Bruker). All spectra were recorded in the range from 4000 to $600 \mathrm{~cm}^{-1}$ with a resolution of $4 \mathrm{~cm}^{-1}$, accumulating 128 scans. In a typical measurement, the sample was gently collocated on the spot of ATR accessory and slowly pressed. To ensure the reproducibility of obtained spectra three samples of each type were measured.

\subsection{Animals}

The animals used for the tests were male C57BL/6J mice ( 8 weeks old, Charles River, Calco, Italy). Animals were group-housed in ventilated cages and had free access to food and water. They were maintained under a 12-hour light/dark cycle (lights on at 8:00 am) at a controlled temperature of $(21 \pm 1)^{\circ} \mathrm{C}$ and relative humidity of $(55 \pm 10) \%$. All procedures performed were carried out in accordance with the guidelines established by the European Communities Council Directive (Directive 2010/63/EU of 22 September 2010) and approved by the National Council on Animal Care of the Italian Ministry of Health. All efforts were made to minimize animal suffering and to use the minimal number of animals required to produce reliable results.

\subsection{UVB induced inflammation}


Before inducing UVB inflammation, the animals were anaesthetized with a mixture of ketamine (10\%) and xylazine (5\%) administered in a single intraperitoneal injection. The dorsal skin was shaved, and mice were covered in a UV opaque material with only the desired portion of skin (an area of approximately $1.5 \mathrm{~cm}^{2}$ ) exposed to a narrowband UVB light source that consisted of TL01 fluorescent tubes (Philips, UK, $\lambda \max =312$ $\mathrm{nm})$ producing an even field of irradiation. The amount of UVB irradiation to which animals were exposed was calculated by using a calibrated meter (IL1400A with SEL240/UVB-1/TD filter, ABLE Instruments \& Controls Ltd, UK) at a distance of $15 \mathrm{~cm}$ from the tubes (equivalent to the mice distance). A maximal dose of $500 \mathrm{mJcm}^{-2}$ was used for all experiments. After UVB irradiation, the exposed area was immediately treated by placing an equal size of the electrospun samples and covered with Tegarderm ${ }^{\mathrm{TM}}$ in order to prevent the mice from removing the dressing. Then, the mice returned to their cages and the nanofiber mats were not removed throughout the study until animals were sacrificed for further experimental end-point determinations. Sham mice followed the same procedures without being exposed to UVB radiation.

\subsection{Cytokine analysis}

To investigate the anti-inflammatory effect of the POCA:PPF fibers, cytokine analysis was performed. Mice ( $\mathrm{n}=5$ per group) were sacrificed at different time points $(24,48$ and 96 hours post-induction), and skin samples from the UVB-exposed and non-exposed animals were removed and stored at $-80{ }^{\circ} \mathrm{C}$ until processing. Each sample was homogenized, subsequently centrifuged and the supernatant isolated and stored at $-80{ }^{\circ} \mathrm{C}$. The expression of cytokines (IL-6 and IL-1 $\beta$ ) was measured using ELISA quantikine kit (R\&D system), according to the manufacturer's instructions. The cytokine concentration was normalized against the total protein content for a given sample, as measured using the bicinchoninic acid (BCA) assay (Thermo Scientific, Rockford, IL, USA).

\subsection{Myeloperoxidase assay}

In order to study the myeloperoxidase (MPO)activity in the skin tissue, the myeloperoxidase assay was followed.[29] Skin samples ( $\mathrm{n}=5$ per group) were homogenized in potassium phosphate buffer $(1 \mathrm{~mL}, 5$ $\mathrm{mM}, \mathrm{pH} 6$ ) and then centrifuged at $21000 \mathrm{rpm}$ for $30 \mathrm{~min}$ at $4{ }^{\circ} \mathrm{C}$. The supernatants were discarded, and the pellets were washed again as described above. For the extraction of MPO, the pellets were suspended in $0.5 \%$ hexadecyltrimethylammonium bromide (Sigma Aldrich) in potassium phosphate buffer (50 mM, pH 6) at a tissue $\mathrm{w} / \mathrm{v}$ ratio of $1: 10$ and frozen at $-40{ }^{\circ} \mathrm{C}$. Three freeze/thaw/sonication cycles were performed. Samples were incubated at $4{ }^{\circ} \mathrm{C}$ for $10 \mathrm{~min}$ and then centrifuged at $12500 \mathrm{rpm}$ for $15 \mathrm{~min}$ at $4{ }^{\circ} \mathrm{C}$. Supernatants were collected, and $7 \mu \mathrm{L}$ of these were allowed to react with $200 \mu \mathrm{L}$ of a solution containing $167 \mathrm{mgL}^{-1}$ of o-dianisidine dihydrochloride (Sigma Aldrich) and $0.0005 \%$ hydrogen peroxide (Sigma Aldrich) in potassium phosphate buffer $(50 \mathrm{mM}, \mathrm{pH} 6)$. The change in absorbance was recorded at intervals of $30 \mathrm{~s}$ for $2 \mathrm{~min}$ at $460 \mathrm{~nm}$ using a microplate reader. One unit of MPO activity was defined as the quantity of enzyme degrading $1 \mu \mathrm{mol}$ of hydrogen peroxide per minute at $25^{\circ} \mathrm{C}$.

\subsection{Histological measurements}

Mice were sacrificed and skin tissue collected (an area of $8 \mathrm{~mm}$ in diameter was excised). The histological specimens were fixed in formalin, embedded in paraffin; $5 \mu \mathrm{m}$ serial sections were collected. Sections were stained with hematoxylin and eosin (H\&E) andacquired with a Leica DMI5500 system. Epidermal thickness and derma cell density were analyzed using ImageJ Software. A $3 \mathrm{~mm}$ length region was randomly selected in each histological section before the measurements. To evaluate epidermal thickness $(\mu \mathrm{m})$, epidermis was underlined, and the thickness was measured drawing a line starting from the underlined area and going 
perpendicularly to the edge of the tissue. At least 20 length measurements for each section were collected. To determine dermal cell density, 5 random dermal areas $\left(0.26 \mathrm{~mm}^{2} \pm 0.05 \mathrm{~mm}^{2}\right)$ were selected. For each region of interest (ROI), area and cell numbers were calculated. The cell density of each ROI was obtained by dividing the cell count with the relative area.

\subsection{Data analysis}

The analysis of the data was presented as means \pm standard error. For ELISA determination, the value obtained from each mouse was calculated as $\mathrm{pg} / \mathrm{mg}$ of protein. Two way ANOVA was used to evaluate statistical significance, followed by Bonferroni's post-hoc test. Two sided Student's t-test was assessed to evaluate statistical differences in epidermal thickness and dermal cell density (*** $\mathrm{p}<0.001)$. GraphPad Prism 5 was used for all statistical analysis (GraphPad Software Inc. San Diego, CA, USA). P values less than 0.05 were considered significant.

\section{Results and discussion}

\subsection{Electrospun fibers characterization}

Fibrous mats were produced by electrospinning a chloroform solution of POCA:PPF $(25 \% \mathrm{wt} / \mathrm{v})$. The two polymers were blended using a ratio of 7:3 (POCA:PPF), because it ensured good electro-spinnability of the final solution. POCA is extensively used as medical adhesive, for instance for the treatment of open wounds and cornea perforations[30-32], and therefore it is already approved for topical uses. Although the direct electrospinning of pure POCA is difficult to achieve, we observed that its combination with PPF allowed the production of well-defined and continuous fibers, as shown in figures $1 \mathrm{a}$ and $1 \mathrm{~b}$. The electrospun mats consisted on a dense network of nanofibers having a regular and cylindrical shape, without the presence of defects or beads. The average diameter was of $(0.8 \pm 0.1) \mu \mathrm{m}$ for the most represented population of fibers (figure 1c). In particular, about $60 \%$ of the fiber population had a diameter in the range of $(0.7-1.1) \mu \mathrm{m}$. In figure $1 \mathrm{~d}$ is shown a photograph of one POCA:PPF fibrous dressing, indicating high flexibility and therefore the possibility to use it for completely covering irregular or curved surfaces. Mechanical tests on the electrospun mats (figure S1 in the Supporting Information) revealed that the fibrous network exhibited a Young's modulus of (2.2 \pm 0.8$) \mathrm{MPa}$, and good elongation at fracture $(0.24 \pm 0.17)$.

(a)

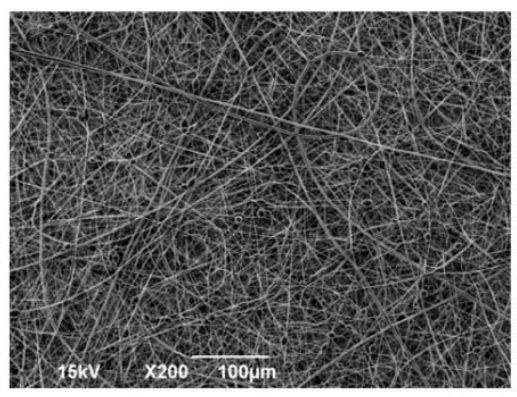

(b)

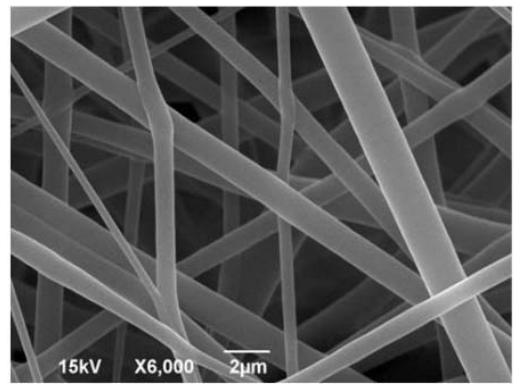

(c)

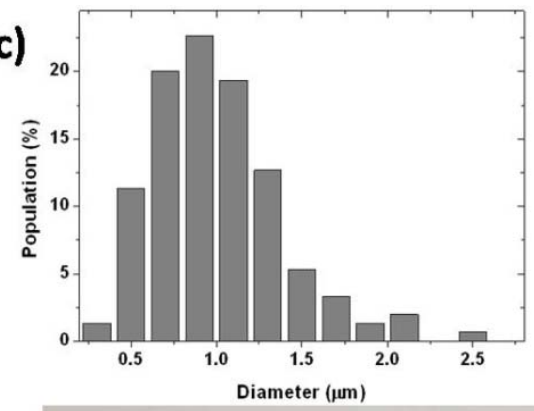

(d)

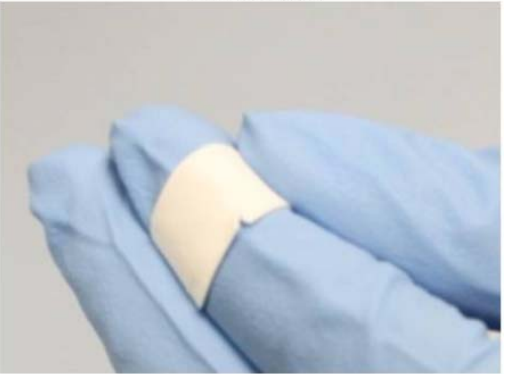


"Figure 1. a) and b) SEM images of POCA:PPF electrospun fibers at different magnifications. c) Analysis of the diameter distribution of the produced fibers. d) Photograph of an electrospun mat used as a bandage".

The chemical properties of the electropun fibers were investigated by attenuated total reflection (ATR) Fourier transform infrared (FTIR) spectroscopy. Figure 2a shows the ATR-FTIR spectra of pure PPF, pure OCA monomer, the gel resultant from the combination of OCA and dimethylacetamide (DMAc) (for the promotion of polymerization of OCA, resulting in gel formation, it was mixed with DMAc at 1:1 ratio (v/v), see experimental section), and finally of POCA:PPF fibers. Absorption peaks associated to pure PPF were: $\mathrm{C}=\mathrm{O}$ stretch at $1715 \mathrm{~cm}^{-1}, \mathrm{C}=\mathrm{C}$ stretch at $1643 \mathrm{~cm}^{-1}, \mathrm{CH}_{2}$ scissoring bend at $1454 \mathrm{~cm}^{-1}, \mathrm{C}-\mathrm{O}-\mathrm{C}$ stretch at 1256 and $1151 \mathrm{~cm}^{-1}$, and $\mathrm{C}-\mathrm{H}$ bend due to the double bond at $978 \mathrm{~cm}^{-1}$.[33] For the OCA monomer several bands associated with functional groups of long-chain cyanoacrylate structure were identified: asymmetrical and symmetrical $\mathrm{CH}_{2}$ stretch at 2928 and $2858 \mathrm{~cm}^{-1}$, respectively, $\mathrm{C} \equiv \mathrm{N}$ stretch in monomer molecular environment at $2237 \mathrm{~cm}^{-1}$, conjugated $\mathrm{C}=\mathrm{O}$ stretch at $1731 \mathrm{~cm}^{-1}, \mathrm{C}=\mathrm{C}$ stretch at $1614 \mathrm{~cm}^{-1}$, and conjugated C-O-C stretching modes at 1286 and $1192 \mathrm{~cm}^{-1}$.[34-36] The infrared spectrum of the OCA-DMAc gel sample displayed absorptions ascribed to DMAc $\left(\mathrm{C}=\mathrm{O}\right.$ stretch at $1641 \mathrm{~cm}^{-1}$, scissoring $\mathrm{CH}_{3}$ bend at $1394 \mathrm{~cm}^{-}$ 1, C-N stretch at $1254 \mathrm{~cm}^{-1}$, and rocking $\mathrm{CH}_{3}$ bend at $1011 \mathrm{~cm}^{-1}$ ) and to the polymer of OCA, named POCA $\left(\mathrm{C} \equiv \mathrm{N}\right.$ stretch in polymer molecular environments at $2249 \mathrm{~cm}^{-1}$ and saturated $\mathrm{C}=\mathrm{O}$ stretch at $1746 \mathrm{~cm}^{-}$ $\left.{ }^{1}\right)$. [36,37,39] This type of polymerization, that converts cyanoacrylate monomers into macromolecules induced by nucleophilic substances such as DMAc, has been previously documented.[29,40] Finally, the composite nanofibers are characterized by absorptions of POCA (in this case, it is observed an additional vibration at $1252 \mathrm{~cm}^{-1}$ related to saturated C-O-C stretch, masked in the gel sample by DMAc bands) and PPF without traces of solvents. The principle spectral differences between the composite nanofibers and the $\mathrm{PPF}$ and POCA samples were located in the region of $\mathrm{C}=\mathrm{O}$ stretch of ester groups (figure $2 \mathrm{~b}$ ). A shift of 15 $\mathrm{cm}^{-1}$ to higher wavenumbers was detected for the carbonyl stretching mode in ester environments of PPF in the case of the composite nanofibers. This is indicative of a transition from a typical situation of 1,4carbonyl-2-ene structures, such as fumarate structures where carbonyl groups can resonate through the double bond, to free saturated ester groups. This situation can be justified considering that double bonds of fumarates are deficient in electrons and can interact with electron-donors as the $\mathrm{N}$ of nitrile functional groups of POCA via dipole-dipole interactions, reducing its effect on carbonyls (figure 2c). In fact, double bonds of fumarates are considered "dipolophiles" in reactions of 1,3-dipolar cycloadditions, while nitrile groups are well-known dipoles.[39,40] 
(a)

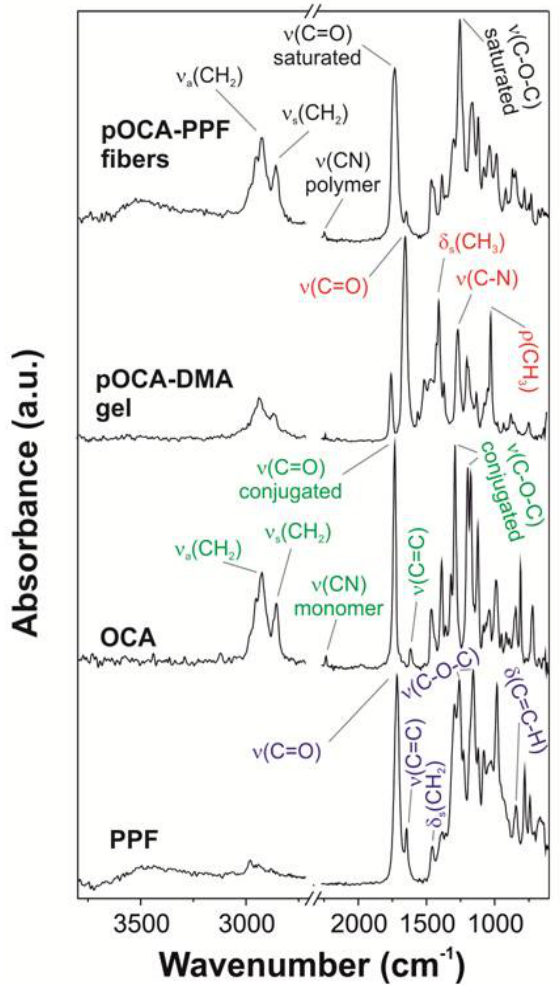

(b)

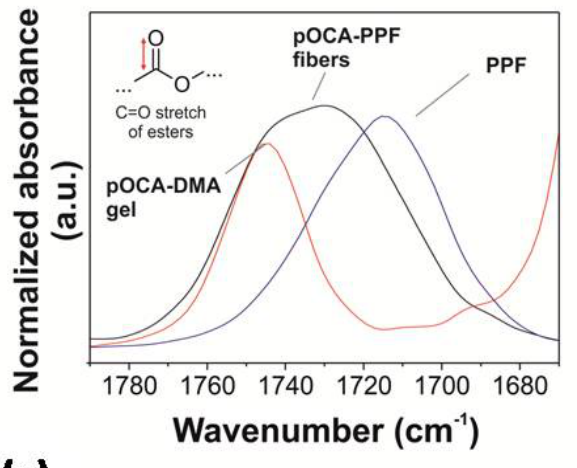

(c)

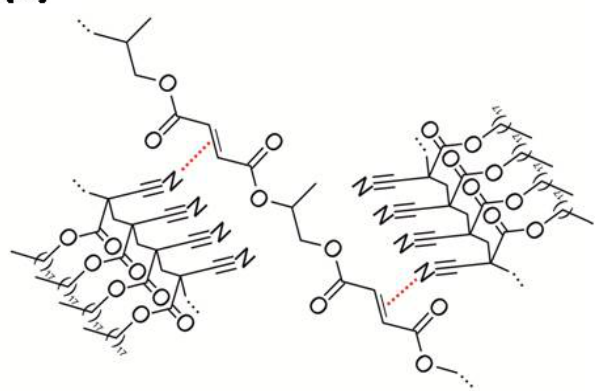

"Figure 2. a) ATR-FTIR spectra of starting materials (PPF and OCA), gel (the product resultant from OCA and DMAc, called POCA) and fiber samples. Main bands associated with PPF (blue color), OCA (green color), DMAc (red color) and POCA (black color) are assigned. b) Comparison of the $\mathrm{C}=\mathrm{O}$ stretching mode of ester groups for pure PPF, POCA in the gel and POCA-PPF fibers. c) Schematic representation of proposed dipole interactions (red dashed lines) between the nitrile groups of POCA and double bonds of PPF."

\subsection{Citokynes and MPO analysis}

POCA and PPF are biocompatible polymers with high potential for regenerative medicine, as it has been reported by other groups.[41] Here, we conducted tests on POCA:PPF fibrous dressings in order to demonstrate their anti-inflammatory activity. Three different groups of mice were investigated: animals not exposed to UVB light (no UVB), exposed to UVB but not treated with dressings (UVB), and exposed to UVB and treated with POCA:PPF dressings (UVB-POCA:PPF). The response of the animals to the injury was investigated by measuring the levels of pro-inflammatory cytokines, the MPO activity and performing histological analysis. In our study, the exposure of the animal skin to UVB irradiation produced evident erythema with signs of mild burn that lead to visible skin lesions and scar formation in 48-96 hours. Redness became apparent after 12-24 hours from the UVB-induced inflammation, but none of the animals developed blisters. We observed a more evident skin injury after 48 hours from the exposure. No edema was observed throughout the test period. Redness and erythema, due to the vasodilation of the cutaneous blood vessels, are typical cutaneous manifestations of the response of the skin to UVB-induced inflammation,[44] and they are characterized by the up-regulation of pro-inflammatory cytokines, together with the release of neuropeptides, histamine, prostaglandins, serotonin and oxygen radicals.[45]

The levels of Interleukin 6 (IL-6) and Interleukin $1 \beta$ (IL-1 $\beta$ ) were analyzed at different time points: 24, 48 and 96 hours after the induction of the inflammation. The expression of IL- 6 and IL-1 $\beta$, measured using 
ELISA quantikine kit and normalized against the total protein, is showed in figure $3 \mathrm{a}$ and $3 \mathrm{~b}$, respectively. As expected, the naive animals expressed a low level of both cytokines, indication that no inflammation was present. On the contrary, these values dramatically increased after the UVB irradiation of the animals' skin, with a peak at 48 hours. Then, a reduction was observed at 96 hours, due to the normal recovery process. When the skin burn was treated with the electrospun POCA:PPF dressings, instead, the detected levels of cytokines significantly decreased. In particular a reduction of about 4 and 14 times was observed for IL- 6 and IL-1 $\beta$ at 48 hours, respectively. Interestingly, the cytokine profiles exhibited a time-constant behavior that could be related to the reduction of the inflammation during the healing process. Moreover, the level of IL-1 $\beta$ was comparable, for all the time points, with the one of the control animals. The obtained results clearly demonstrate that the electrospun POCA:PPF fibers possess anti-inflammatory activity and they effectively promote the healing of UVB-induced burns.

As previously discussed, fumarate-based materials have been already used for their ability to treat inflammatory states.[14,17]However, electrospun fibres of those materials have not been reported yet for the treatment of skin burns. Nanofibers made of or loaded with natural products have been instead investigated, such as silk fibroin, alginate, essential oils, honey and other organic components with anti-inflammatory activity. $[11,46,47]$ The use PPF for the production of electrospun dressings avoids the loading of the fibres with anti-inflammatory drugs, because PPF itself works both as structural component of the fibres and as active agent.

In order to strengthen the hypothesisthat the anti-inflammatory properties of the produced POCA:PPFfibers are ascribed to the presence of PPF, we fabricated POCA fibers in which PPF was substituted with polycaprolactone (PCL). We adopted this strategy since it was not possible to produce pure PPF fibers due to its low electrospinnability. PCL was chosen because it is a polyester as PPF, but it is biologically inert. We analyzed in-vivo the cytokine expression for UVB-exposed animals treated with POCA:PCL dressings. In Figure S2 (Supporting Information) the cytokine levels at 48 hours are reported. This time point was chosen because it represented the peak of cytokines expression for UVB-exposed untreated animals. By comparing the data obtained for mice treated with POCA:PPF and POCA:PCL fibers it is clearly visible that POCA:PCL dressings were ineffective in limiting cytokines expression. In fact, no statistically significant differences were observed be-tween the group treated with POCA:PCL mats and the untreated one. Therefore, we can safely conclude that PPF is responsible for the anti-inflammatory activity of the produced nanofibrous dressings.

The analysis of the MPO activity for the three groups of animals (no UVB, UVB and UVB-POCA:PPF) was conducted to further validate the anti-inflammatory properties of the electrospun dressings. MPO is a plentiful constituent of neutrophils and it is used as a marker for tissue neutrophils recruitment to the wound site during the healing process. The comparison of the results at 24, 48 and 96 hours is shown in Figure 3c. Similarly to cytokine expression profile, for UVB-exposed mice the MPO activity had a peak at 48 hours and then decreased at 96 hours. This indicates that the neutrophils were activated at 48 hours in response to inflammatory mediators, such as cytokines. On the contrary, MPO values strongly decreased when the burn was covered with the POCA:PPF fibers. A comparison at 48 hours, between the MPO value for animals treated with PPF-loaded fibers and that recorded for untreated mice, revealed a reduction by approximately three times in the first case (figure 3c). 


\section{(a)}

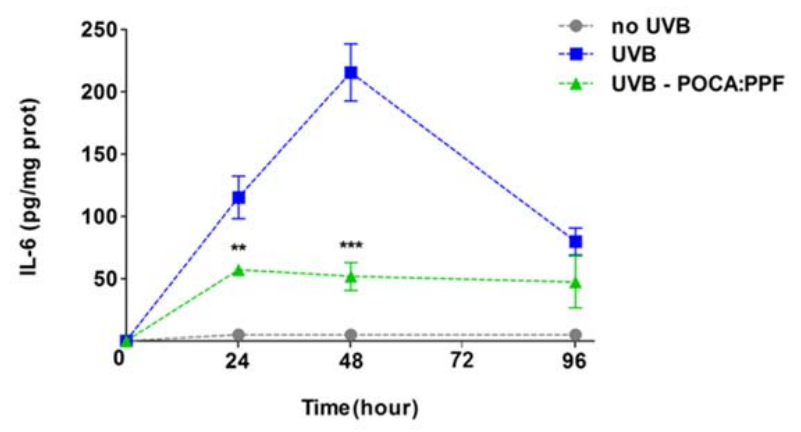

(b)

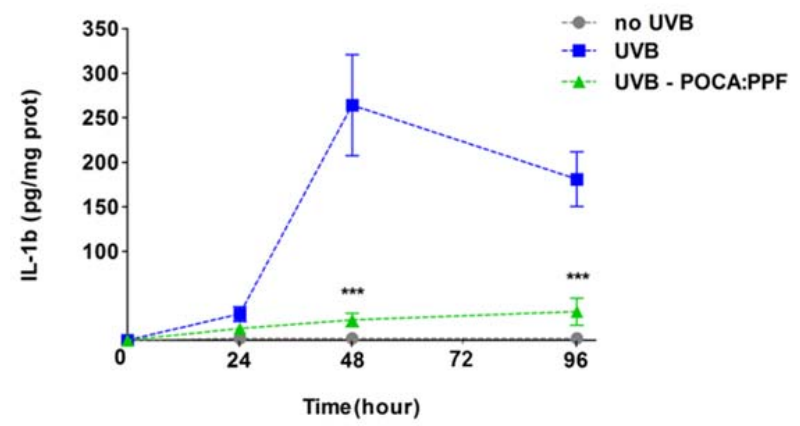

(c)

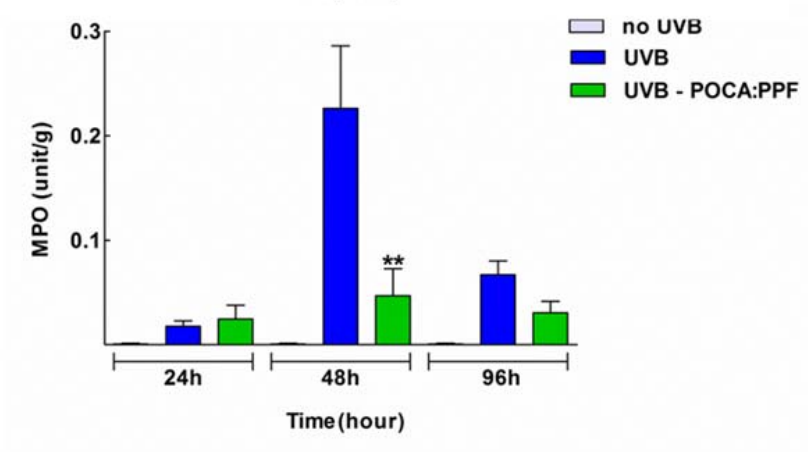

"Figure 3. a-b) Time course of the expression of IL-6 and IL-1 $\beta$, respectively, for control animals (grey symbols), mice ex-posed to UVB light (blue symbols) without and (green symbols) with POCA:PPF treatment. c) Time course of the MPO activity of skin tissues of the same groups of animals. For all the panels, each value is the average \pm stand. error of five animals per group after 24,48 and 96 hours from the injury. ${ }^{* * *} \mathrm{p}<0.001,{ }^{* *} \mathrm{p}<0.01$ vs. UVB irradiated group. Two-way ANOVA, post hoc Bonferroni test.”

\subsection{Histological analysis}

In order to confirm the healing effect of the PPF-loaded nanofibers, histology tests were conducted on animals'skin after 48 hours from the induced inflammation (peak of inflammatory phase). The histology was performed on samples from naïve animals (no UVB), from mice irradiated (UVB), and from animals irradiated and treated with POCA:PPF fibrous dressings (UVB-POCA:PPF). The microanatomy of the skin of a control animal (figure $4 \mathrm{a}$ and $4 \mathrm{~d}$ ) shows a thin external epithelium (epidermis) formed by two to three cell layers, a central thick layer of connective tissue (dermis), and a innermost layer of adipose tissue (hypodermis or panniculus adiposus). A thin layer of striated muscle, known as panniculus carnosus, separates the skin from other structures. After the UVB irradiation, an acute inflammation was evident, characterized by infiltration of neutrophils and epithelial hyperplasia (figure $4 \mathrm{~b}$ and $4 \mathrm{e}$ ). The latter is the most common spontaneous, non-neoplastic lesion of the skin that is observed in a wide variety of injuries and conditions, including experimentally induced inflammatory processes, exposure to irritant materials, and 
prolonged exposure to UV light.[48]In the UVB exposed mice, the epithelial hyperplasia determined the

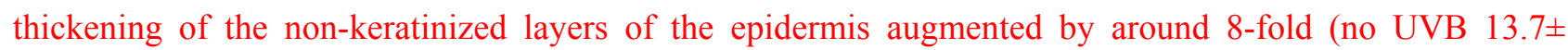
$1.4 \mu \mathrm{m} v \mathrm{~s}$. UVB $106.4 \pm 37.9 \mu \mathrm{m}$, figure $4 \mathrm{~g}$ ) due to an increased number (layers) of new epithelial cells compared to the ones at the same site in a control animal. Indeed ulcers, mild to marked, were observed in all sections of burned skin. Furthermore, wounded tissues showed an increase of 2.5 -fold in derma cell number (no UVB $1100 \pm 112$ cells $/ \mathrm{mm}^{2}$ vs. UVB $2792 \pm 544 \mathrm{cells} / \mathrm{mm}^{2}$ ) as results of inflammatory process (figure $4 \mathrm{~h}$ ). When the UVB-induced wound was treated with the POCA:PPF dressings (figure $4 \mathrm{c}$ and $4 \mathrm{f}$ ), the severity of the inflammation was reduced to a few minimal and local focalization, and the skin appeared similar to that one of naïve animals in term of skin thickness (no-UVB 13.7 $\pm 1.4 \mu \mathrm{m} v s$. UVB POCA:PPF $14.3 \pm 2.5 \mu \mathrm{m}$ ) and mildly more infiltrated (no-UVB $1100 \pm 112$ cells $/ \mathrm{mm}^{2} v$ s. UVB POCA:PPF $1358 \pm$ $255 \mu \mathrm{m}$, figure $4 \mathrm{~g}$ and $\mathrm{h}$ ). Indeed, a marked reduction by about $99 \%$ of the epidermis thickness and by about $85 \%$ in derma cell density was observed compared to the injured untreated animals, demonstrating the suitability of the dressing in accelerating and effectively promoting the burn healing process.

(a)

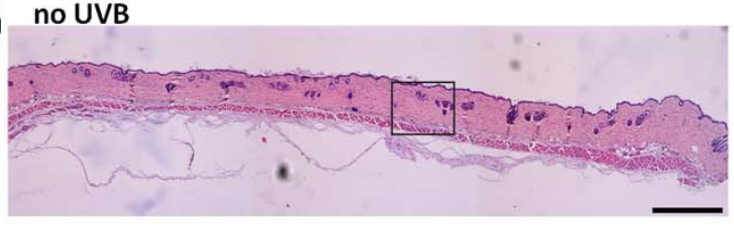

(b)

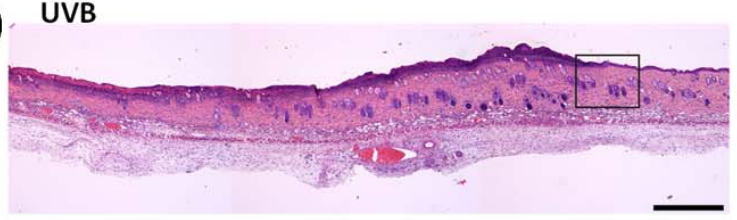

(c)

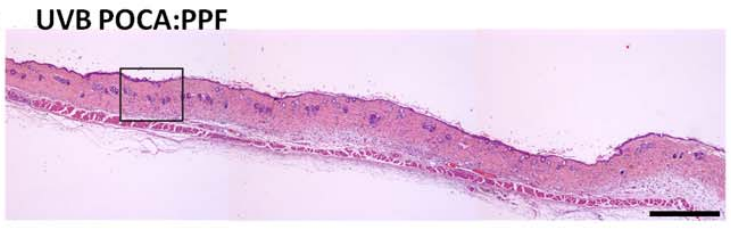

(d)

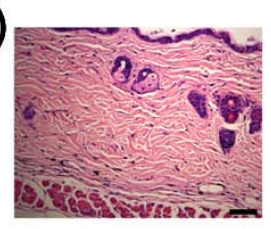

(e)

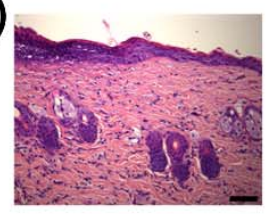

(f)

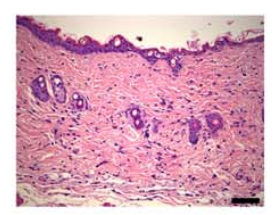

(g)

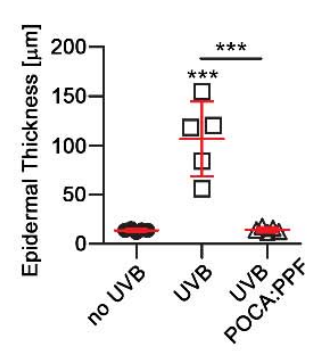

(h)

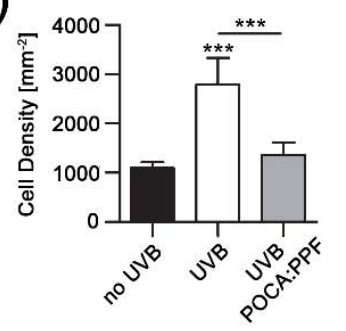

"Figure 4. Selection of images of H\&E stained skin sections for animals a,d) not injured, b,e) exposed to UVB light but untreated, and c,f) injured and treated with POCA:PPF dressings, and histological measurements of g) epidermal thickness and h) derma cell density ( $\mathrm{n}=5$ mice per group). Scale bars of $500 \mu \mathrm{m}$ for a), b), c), and of $50 \mu \mathrm{m}$ for d), e), f)."

\section{Conclusion}

In conclusion, we produced novel wound dressings by electrospinning poly(octyl cyanoacrylate) combined with polypropylene fumarate, where the latter acts as anti-inflammatory drug. The electrospinning process was optimized and the fibers were characterized in terms of morphological, mechanical and chemical properties. Their anti-inflammatory activity was tested in-vivo on UVB-irradiated animals. These studies indicated that the presence of the fumarate promoted the reduction of cytokines production and allowed low cytokines production and neutrophils' levels 48 hours after the induced burn. Moreover histological analysis 
suggested that the skin treated with POCA:PPF fibers recovered faster its healthy appearance characterized by a thin epidermal layer and a limited number of inflammatory cells. We believe that the developed nanofibrous mats, where for the first time was employed the polypropylene fumarate, could be ideal dressings for burn wounds since they accelerate the healing process, restoring the original skin conditions.

\section{Acknowledgment}

José A. Heredia-Guerrero is supported by a Marie Curie In-tra-European Fellowship (BIOPROTO project), financed by the EU's Seventh Framework Programme for Research (FP7).

\section{References}

[1] Schneider A, Wang X Y, Kaplan D L, Garlick J A and Egles C 2009 Biofunctionalized electrospun silk mats as a topical bioactive dressing for accelerated wound healing. Acta Biomater.5 2570-8

[2] Chen J-P, Chang G-Y and Chen J-K 2008 Electrospun collagen/chitosan nanofibrous membrane as wound dressing Colloids Surfaces A Physicochem. Eng. Asp.313-314 183-8

[3] Park S A, Teixeira L B C, Raghunathan V K, Covert J, Dubielzig R R, Isseroff R R, Schurr M, Abbott N L, McAnulty J and Murphy C J 2014 Full-thickness splinted skin wound healing models in $\mathrm{db} / \mathrm{db}$ and heterozygous mice: implications for wound healing impairment. Wound Repair Regen.22 $368-80$

[4] Hamed S, Bennett C L, Demiot C, Ullmann Y, Teot L and Desmoulière A 2013 Erythropoietin, a novel repurposed drug: an innovative treatment for wound healing in patients with diabetes mellitus. Wound Repair Regen.22 23-33

[5] Shan Y-H, Peng L-H, Liu X, Chen X, Xiong J and Gao J-Q 2015 Silk fibroin/gelatin electrospun nanofibrous dressing functionalized with astragaloside IV induces healing and anti-scar effects on burn wound. Int. J. Pharm.479 291-301

[6] Jeschke G. Marc, Shahriar Shahrokhi, Finnerty C. Celeste, Branski K. Ludwuik D M 2014 Wound Coverage Technologies in Burn Care: Established Techniques J. Burn Care Res. 1-7

[7] Jayme A Farina Jr, Rosique M J and Rosique R G 2013 Curbing Inflammation in Burn Patients Int. J. Inflam.2013 1-9

[8] Zahedi P, Rezaeian I, Ranaei-Siadat S-O, Jafari S-H and Supaphol P 2009 A review on wound dressings with an emphasis on electrospun nanofibrous polymeric bandages Polym. Adv. Technol. n/a $-\mathrm{n} / \mathrm{a}$

[9] Rieger K a., Birch N P and Schiffman J D 2013 Designing electrospun nanofiber mats to promote wound healing - a review J. Mater. Chem. B1 4531

[10] Mogoşanu G D and Grumezescu M A 2014 Natural and synthetic polymers for wounds and burns dressing 463 127-36

[11] Hadi Hajiali, Maria Summa, Debora Russo, Andrea Armirotti, Virgilio Brunetti, Rosalia Bertorelli, Athanassia Athanassiou E M 2016 Alginate-lavender nanofibers with antibacterial and antiinflammatory activity to effectively promote burn healing J. Mater. Chem. B4 1686-95

[12] Mrowietz U and Asadullah K 2005 Dimethylfumarate for psoriasis: more than a dietary curiosity. Trends Mol. Med.11 43-8

[13] Lin S X, Lisi L, Dello Russo C, Polak P E, Sharp A, Weinberg G, Kalinin S and Feinstein D L 2011 
The anti-inflammatory effects of dimethyl fumarate in astrocytes involve glutathione and haem oxygenase-1. ASN Neuro3 75-84

[14] Gill A J and Kolson D L 2014 Dimethyl fumarate modulation of immune and antioxidant responses : application to HIV therapy Crit Rev Immunol33 307-59

[15] Rubant S a, Ludwig R J, Diehl S, Hardt K, Kaufmann R, Pfeilschifter J M and Boehncke W-H 2008 Dimethylfumarate reduces leukocyte rolling in vivo through modulation of adhesion molecule expression. J. Invest. Dermatol.128 326-31

[16] H. Jorn Bovenschen, Annechien M.G. Langewouters P C M van de K 2010 Dimethylfumarate for Psoriasis Pronounced Effects on Lesional T-Cell Subsets, Epidermal Proliferation and Differentiation, but not on Natural Killer T Cells in Immunohistochemical Study Arn J Clin Dermatol11 343-50

[17] Linker R a and Gold R 2013 Dimethyl fumarate for treatment of multiple sclerosis: mechanism of action, effectiveness, and side effects. Curr. Neurol. Neurosci. Rep.13 394

[18] Beke S, Farkas B, Romano I and Brandi F 2012 Mask Projection Excimer laser Stereolithography : a scalable method for 3D biodegradable scaffold fabrication Opt. Mater. Express015001

[19] Beke S, Anjum F, Ceseracciu L, Romano I, Athanassiou a, Diaspro a and Brandi F 2013 Rapid fabrication of rigid biodegradable scaffolds by excimer laser mask projection technique: a comparison between 248 and $308 \mathrm{~nm}$ Laser Phys.23 035602

[20] Kasper F K, Tanahashi K, Fisher J P and Mikos A G 2009 Synthesis of poly(propylene fumarate). Nat. Protoc.4 518-25

[21] Wei C, Cai L, Sonawane B, Wang S and Dong J 2012 High-precision flexible fabrication of tissue engineering scaffolds using distinct polymers. Biofabrication 4025009

[22] Jayabalan M, Thomas V and Rajesh P N 2001 Polypropylene fumarate/phloroglucinol triglycidyl methacrylate blend for use as partially biodegradable orthopaedic cement. Biomaterials22 2749-57

[23] Kim C W, Talac R, Lu L, Moore M J, Currier B L and Yaszemski M J 2008 Characterization of porous injectable poly-(propylene fumarate)-based bone graft substitute. J. Biomed. Mater. Res. A85 $1114-9$

[24] Dominique G. Poituit 2004 Biomechanism and biomaterials in Orthopedics

[25] Ueda H, Hacker M C, Haesslein A, Jo S, Ammon D M, Borazjani R N, Kunzler J F, Salamone J C and Mikos A G 2007 Injectable, in situ forming poly(propylene fumarate)-based ocular drug delivery systems J. Biomed. Mater. Res. Part A83A 656-66

[26] Hacker M C, Haesslein a, Ueda H, Foster W J, Garcia C a, Ammon D M, Borazjani R N, Kunzler J F, Salamone J C and Mikos a G 2009 Biodegradable fumarate-based drug-delivery systems for ophthalmic applications. J. Biomed. Mater. Res. A88 976-89

[27] Jiang K, Long Y-Z, Chen Z-J, Liu S-L, Huang Y-Y, Jiang X and Huang Z-Q 2014 Airflow-directed in situ electrospinning of a medical glue of cyanoacrylate for rapid hemostasis in liver resection. Nanoscale6 7792-8

[28] S. G. Kumbar, C. T. Laurencin M D 2014 Natural and Synthetic biomedical polymers

[29] Mele E, Heredia-Guerrero J A, Bayer I S, Ciofani G, Genchi G G, Ceseracciu L, Davis A, Papadopoulou E L, Barthel M J, Marini L, Ruffilli R and Athanassiou A 2015 Zwitterionic Nanofibers of Super-Glue for Transparent and Biocompatible Multi-Purpose Coatings Sci. Rep.5 14019

[30] Lee J W, Ahn G, Kim D S and Cho D-W 2009 Development of nano- and microscale composite 3D 
scaffolds using PPF/DEF-HA and micro-stereolithography Microelectron. Eng.86 1465-7

[31] Couturier J Y, Ding-Zhou L, Croci N, Plotkine M and Margaill I 2003 3-Aminobenzamide reduces brain infarction and neutrophil infiltration after transient focal cerebral ischemia in mice. Exp. Neurol.184 973-80

[32] Wachter D, Brückel A, Stein M, Oertel M F, Christophis P and Böker D-K 2010 2-Octylcyanoacrylate for wound closure in cervical and lumbar spinal surgery. Neurosurg. Rev.33 483-9

[33] Okabe M, Kitagawa K, Yoshida T, Koike C, Katsumoto T, Fujihara E and Nikaido T 2013 Application of 2-octyl-cyanoacrylate for corneal perforation and glaucoma filtering bleb leak Clin. Ophthalmol.5 649-53

[34] Torres R, Christina R, Gomes B and Vanessa P 2012 Use of cyanoacrylate in the coaptation of edges of surgical An Bras Dermatol87 871-6

[35] Peter SJ., Yaszemski MJ., Suggs LJ., Payne RG., Langer R., Hayes WC., Unroe MR., Alemany LB., Engel PS. M A 1997 Characterization of partially saturated poly(propylene fumarate) for orthopaedic application . Biomater. Sci. Polym. Edn8 893-904

[36] Edwards H G M and Day J S 2004 Fourier transform Raman spectroscopic studies of the curing of cyanoacrylate glue J. Raman Spectrosc.35 555-60

[37] Liu S.X., Han L.M.G., Kim S. 2008 Synthesis and degradation behavior of poly(ethyl cyanoacrylate). Polym. Degrad. Stab.93 1243-51

[38] Bellamy L 1975 The Infrared Spectra of Complex Molecules ed C and Hall (New York)

[39] Dwivedi, AM., Krimm S. M S 1989 Vibrational force field and normal mode analysis of N,Ndimethylacetamide. Spectrochim. Acta45A 271-9

[40] Donnelly EF., Johnston DS., Pepper DC. D D 1977 Ionic and zwitterionic polymerization of n-alkyl 2-cyanoacrylates. Polym. Lett. Ed.15 399-405

[41] Gholami MR. H Y A 2000 Kinetics of 1, 3-dipolar cycloaddition reaction between C, Ndiphenylnitrone and dimethyl fumarate in various solvents and aqueous solutions. Int. J. Chem. Kinet.32 431-4

[42] Cumper CWN., Dev SK. L S 1973 Electric dipole moments of some acrylonitriles, allyl cyanides, and alicyclic nitriles. J. Chem. Soc.2 537-40

[43] Nair L S and Laurencin C T 2006 Polymers as biomaterials for tissue engineering and controlled drug delivery. Adv. Biochem. Eng. Biotechnol.102 47-90

[44] Clydesdale G J, Dandie G W and Muller H K 2001 Ultraviolet light induced injury: Immunological and inflammatory effects Immunol. Cell Biol.79 547-68

[45] Saadé N E, Farhat O, Rahal O, Safieh-Garabedian B, Bars D Le and Jabbur S J 2008 Ultra violetinduced localized inflammatory hyperalgesia in awake rats and the role of sensory and sympathetic innervation of the skin Brain. Behav. Immun.22 245-56

[46] Chae M, Hyeon S, Ren J, Seok C and Hum C 2016 Wound healing effect of electrospun silk fibroin nanomatrix in burn-model Int. J. Biol. Macromol.85 29-39

[47] Claassen L, Papst S, Reimers K, Steinstraesser L, Vogt P M, Kraft T and Niederbichler A D 2014 Inflammatory Response to Burn Trauma : Nicotine 6 392-401

[48] Papp A, Kiraly K, Härmä M, Lahtinen T, Uusaro A and Alhava E 2004 The progression of burn depth in experimental burns : a histological and methodological study Burns30 684-90 
